\title{
The Micromegas chambers for the upgrade of the forward muon detector of ATLAS
}

\author{
Jérôme Samarati*, on the behalf of the ATLAS Muon Collaboration ${ }^{\dagger}$ \\ CERN \\ E-mail: jerome.samarati@cern.ch
}

\begin{abstract}
The ATLAS Collaboration at the LHC has chosen the resistive Micromegas technology, along with the small-strip Thin Gap Chambers, for the high luminosity upgrade of the first muon station in the high- $\eta$ region (i.e. $1.3<|\eta|<2.7$ ), the so called New Small Wheel project. After the R\&D phase, design and prototyping phase, the first series production Micromegas quadruplets are being constructed at the involved construction sites (France, Germany, Italy, Russia and Greece). All the Micromegas boards produced in industries and delivered at CERN are undergoing a detailed quality control and quality assurance under the supervision of the CERN group. This is a big step forward towards the installation of the New Small Wheel foreseen for the LHC long shutdown in 2019 and 2020. The construction of the four types of large size quadruplets, all having trapezoidal shapes with surface areas between 2 and $3 \mathrm{~m}^{2}$, will be briefly described. The achievement of the requirements for these detectors revealed to be even more challenging than expected, when scaling from the small prototypes to the large dimensions. The encountered problems and the proposed solutions are to a large extent common to other micro-pattern gaseous detectors. The final quality on the achieved mechanical precision, and on the high-voltage stability, are described together, with results from test-beam studies with the first production chambers.
\end{abstract}

European Physical Society Conference on High Energy Physics - EPS-HEP2019 -

10-17 July, 2019

Ghent, Belgium

\footnotetext{
* Speaker.

${ }^{\dagger}$ now at the European Spallation Source
} 


\section{Introduction}

The ATLAS [1] experiment is one of the four LHC experiments and it will enter in the first phase of its upgrade. The major upgrade of the detector, presented in Figure 1, will be the replacement of the two innermost forward detector wheels of the ATLAS Muon system by the New Small Wheels (NSW) [2]. Each wheel comprises Micromegas and small Thin Gap Chamber (sTGC) detectors in an overlapping structure of eight large and eight small sectors. Each sector hosts two wedges ( 4 detection layers each) of each technology, for a total of 16 active layers in the full thickness. The Micromegas of the NSWs will cover an active area of $1280 \mathrm{~m}^{2}$, divided in 128 detectors of 4 different module types (the small modules type 1 and 2 for respectively for SM1 and SM2 and the large modules type 1 and 2 respectively for LM1 and LM2) and comprise in total more than $2 \times 10^{6}$ channels. The NSWs shall contribute to a muon $p_{\mathrm{T}}$ measurement with $10 \%$ resolution at 1 $\mathrm{TeV}$ (offline) and will improve the Level 1 trigger (online) resolution.

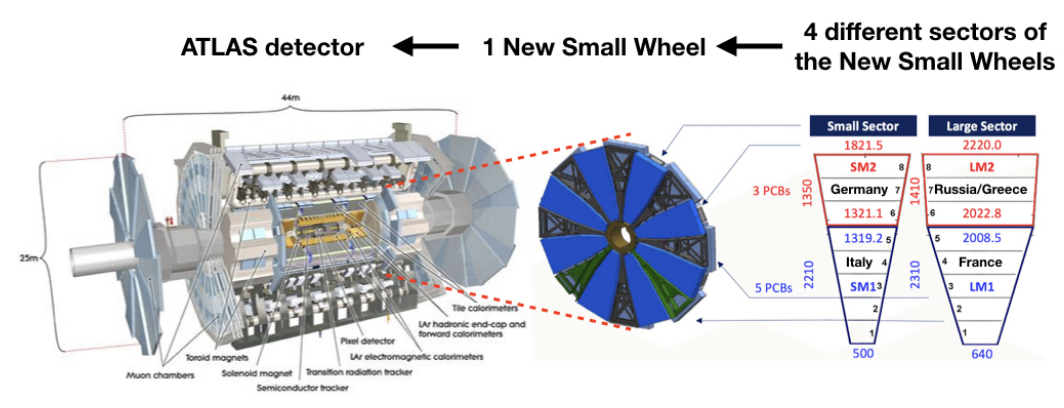

Figure 1: Description of the 4 different types of Micromegas modules composing the New Small Wheels in the ATLAS detector.

The principle of the Micromegas detector is presented in Figure 2. Its geometry consists in a $5 \mathrm{~mm}$ drift gap separated from an amplification gap of $128 \mu \mathrm{m}$ by a very thin metallic mesh (wire diameter of $18 \mu \mathrm{m}$ with an opening of $45 \mu \mathrm{m}$ ). The height of the amplification gap, which is a crucial parameter, is defined very accurately by the height of the pillars (columns of insulating material) that encapsulate the mesh. The anode readout is composed of $\mathrm{Cu}$ strips of $300 \mu \mathrm{m}$ width with a pitch of 425 or $450 \mu \mathrm{m}$ covered by an insulating layer on which resistive strips lie (with the same parameters as for the $\mathrm{Cu}$ ones). A strip resistivity of $\sim 10 \mathrm{M} \Omega / \square$ has been chosen in order to limit drastically the effect of discharges, keeping a rate capability in agreement with the ATLAS requirements.

The Micromegas detectors that will compose the sector are made of four detection layers each ( 2 precision layers called $\eta$ layers and 2 stereo with the readout strips tilted by respectively $+1.5^{\circ}$ and $-1.5^{\circ}$ ) and are called quadruplets. The quadruplet assembly scheme is described in Figure 2. It is composed of a central double sided drift electrode, 2 double sided readout boards and 2 single sided cathode boards. This assembly of those different part leads to a very challenging list of requirements from a technical and mechanical point of view :

- Strip alignment precision of $30 \mu \mathrm{m}$ in the precision coordinate $(\eta)$;

- Planarity with an RMS within $100 \mu \mathrm{m}$; 

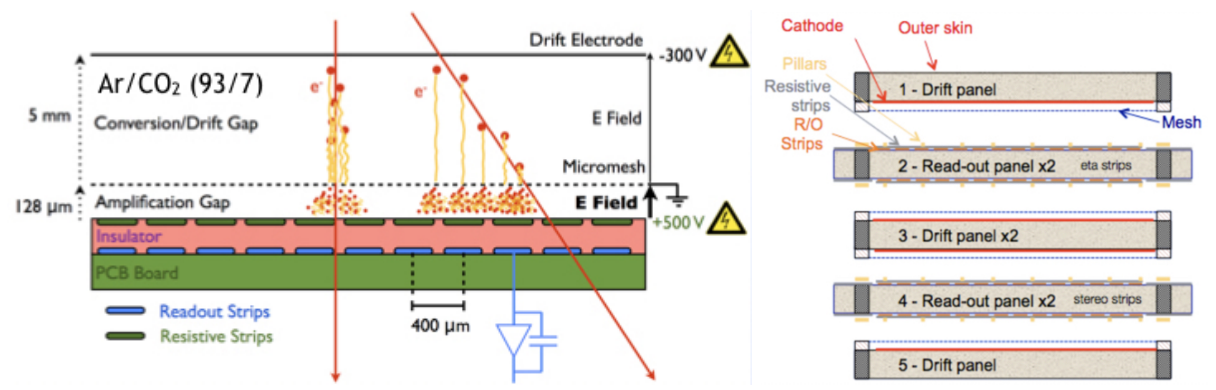

Figure 2: Micromegas principle (left) [3] and the scheme of the assembly of a quadruplet (right).

- A very challenging technological transfer of the read-out boards production to the industry since it requires an extremely high level of quality (pillar shape, resistivity homogeneity, quality of the printed circuit board edges);

- Gas tightness (ATLAS acceptance limits $(1 / \mathrm{h}): 0.023$ for SM1, 0.026 for SM2, 0.038 for LM1 and 0.037 for LM2);

- Stability against discharges under a high electric field $(\mathrm{E} \sim 50 \mathrm{kV} / \mathrm{cm})$ over a surface of the order of $\mathrm{m}^{2}$;

- High voltage stability criteria : nominal voltage $570 \mathrm{~V}$ applied on the resistive strips $(\leq 10$ $\mathrm{nA}$ without irradiation) with $\leq 1$ spike/min.

\section{Results of the commissioning measurements}

In this section, some of the results obtained for each of the items listed below are reported.

\subsection{Strip alignment measurement}

This measurement is realised by measuring the position of the masks located on the readout board using a camera fixed on a CMM (Coordinate Measurement Machine). Figure 3 shows a readout board with its mask. This method allows us to know the absolute value of the alignment for the strips inside a readout board within $40 \mu \mathrm{m}$ and a relative value of the alignment of the planes between themselves within $60 \mu \mathrm{m}$.

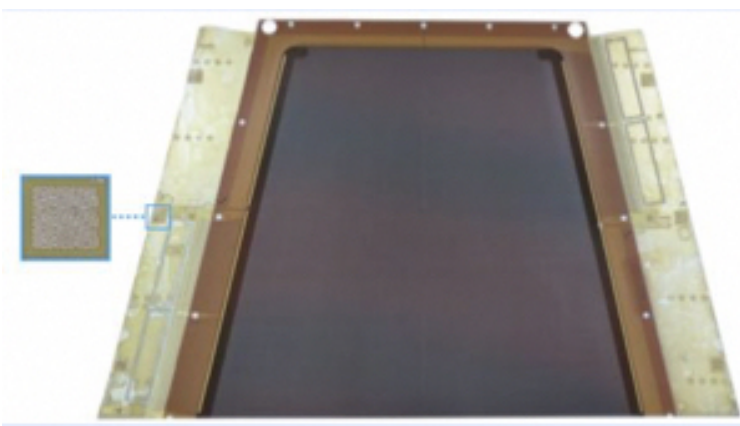

Figure 3: Readout board with the mask used for the alignment measurement. 


\subsection{Planarity measurement}

This measurement consists in measuring the planarity of each panel and also of the quadruplet when mounted, to ensure the strip positioning for each plane in the beam direction. A result of this measurement is presented in Figure 4 and shows a thickness value of $76.91 \mathrm{~mm}$ with a dispersion (RMS) of $\sim 90 \mu \mathrm{m}$ (it should be below $100 \mu \mathrm{m}$ ).

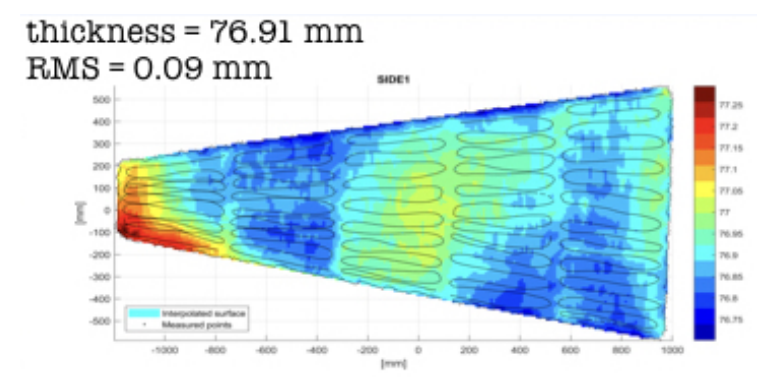

Figure 4: Planarity measurement result done on an SM1 production module in Italy (Pavia).

\subsection{Gas leak measurement}

In order to ensure a correct operation of the detector, the gaseous mixture inside the chamber has to be the one specified in the requirement. This means that each quadruplet has to be gas tight. For this purpose, a very powerful system has been developed based on two mass flow sensors, two differential pressure sensors and one temperature sensor. The system allows us to measure a leak in four chambers simultaneously by two different methods, the Flow Rate Loss (FRL, for which one measure the difference between the inlet and the outlet flow) or the Pressure Drop Rate (PDR for which one measures the time taken for a pressure drop). This is coupled with a software that provides an online monitoring of the leak. The scheme of the system and the results provided for the SM1 are presented in Figure 5.

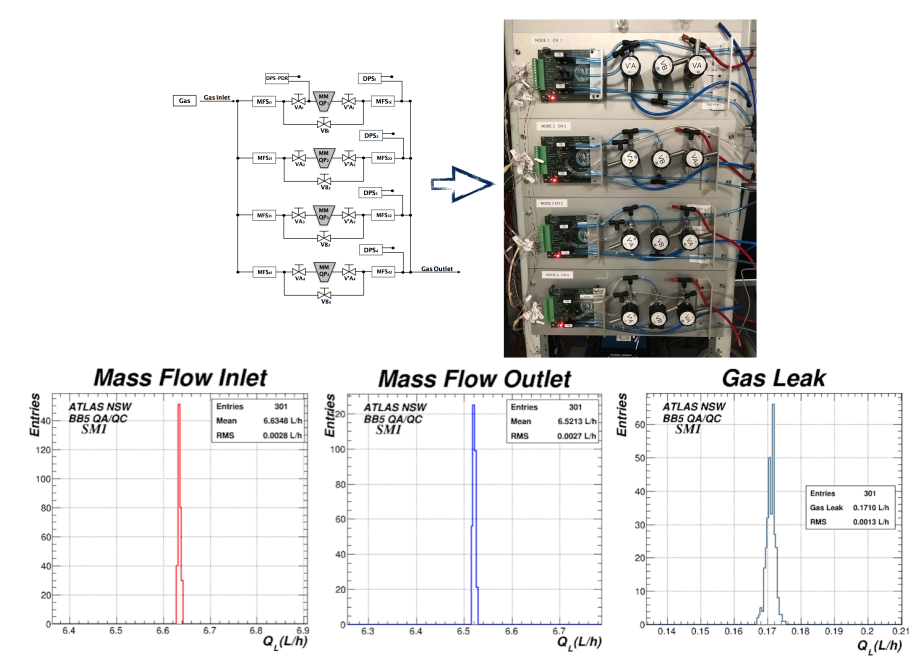

Figure 5: Gas leak test scheme (top) and the FRL method result (bottom). 
The leak value obtained of $0.171 \mathrm{l} / \mathrm{h}$ is well above the acceptance limit, which is $0.023 \mathrm{l} / \mathrm{h}$ for this type of quadruplet. This problem occurred on a pre-production module and has been solved by modifying the assembly procedure, especially the gluing procedure of the panels (readout and drift).

\subsection{High voltage test}

The purpose of this test is to check if the quadruplet can handle its nominal voltage (570 V) but also to check if the spark rate is $\leq 1 \mathrm{spike} / \mathrm{min}$ or if the current at the nominal voltage is $\leq 10 \mathrm{nA}$ without irradiation. We have unfortunately observed that a large fraction of the produced quadruplet do not fullfill the requirements presented in the list below. In Figure 6, we present the curve of the voltage as a function of time for two quadruplets, one that matches the acceptance criteria and a second one that does not.
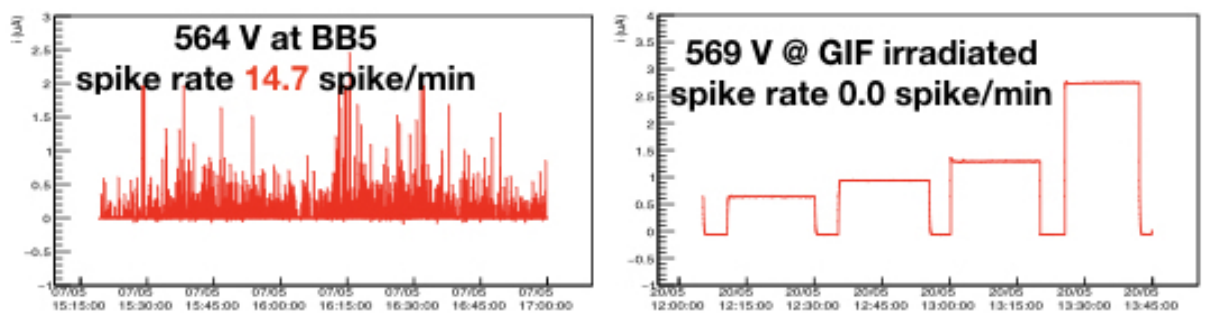

Figure 6: 2 quadruplets : agrees with the acceptance criteria (left) and does not agree the acceptance criteria (right).

In order to understand and to solve this high voltage issue, we have launched a series of tests of several factors :

- Possible defect in the PCB $\rightarrow$ solved (QAQC procedure is well defined to chase any defect of the board);

- Possible defect in the mesh $\rightarrow$ solved (mesh polishing to remove the imperfections);

- Level of humidity inside the detector too high $\rightarrow$ solved (flush the detectors for several hours to dry it);

- Level of cleanliness during the assembly phase not good enough $\rightarrow$ actions now being taken to improve the cleanliness level in all sites to the required level;

- Point of low resistance in the resistive layer are a major concern because they are much less protected in case of discharge $\rightarrow$ actions taken to reduce this effect.

\subsection{Performance of the detectors}

In order to characterise the quadruplets produced in the different construction sites, each detector will undergo a cosmic ray test to measure its efficiency for minimum ionising particles. In Figure 7, we present some results obtained for the German module (SM2).

At the nominal voltage, the detector shows an efficiency of $\geq 90 \%$ for layer 1 and also for the full quadruplet. It also presents a homogeneous response over the whole area of layer 1. 

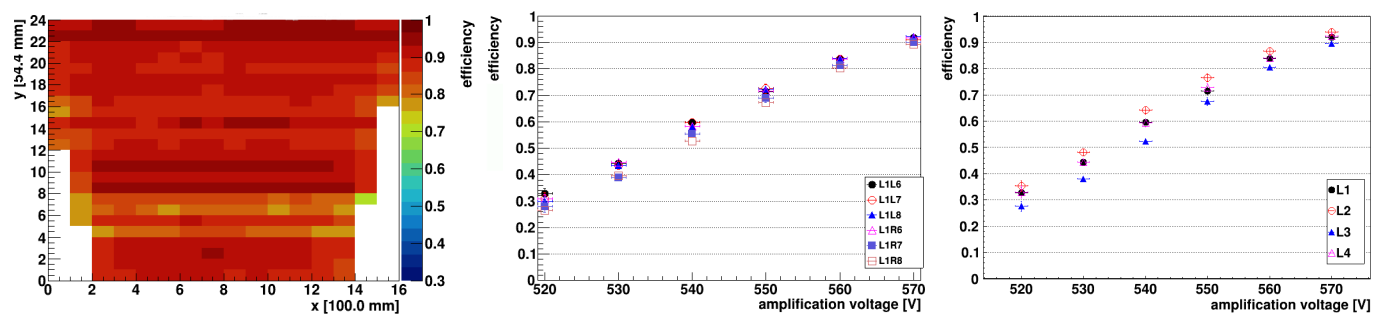

Figure 7: Resulting plots of SM2 : Efficiency map of layer 1 (left), efficiency curve vs high voltage for layer 1 (center) and efficiency curve vs high voltage for the quadruplet (right).

\section{Conclusions}

The delivery of the detectors from the construction sites began a few months ago and has almost reached its cruising speed. The sector assembly has also started with the production of the $1^{\text {st }}$ small sector of the ATLAS Muon spectrometer which allows us to test the full chain of the assembly but also the full procedure of the commissioning of the delivered detectors. The $1^{\text {st }}$ large sector has been assembled few weeks ago (at the time of the writing). This has allowed the assembly procedure to be tested for a large sector. The HV instability should still be investigated in order to be fully understood, so as to avoid major issues during the Run 3 of the LHC.

\section{References}

[1] ATLAS Collaboration, 2008 JINST 3 S08003.

[2] ATLAS Collaboration: ATLAS New Small Wheel Technical Design Report, CERN-LHCC-2013-006, ATLAS-TDR-020-2013, Geneva (2013).

[3] B. Alvarez et al., Radiation studies on resistive bulk-Micromegas chambers at the CERN Gamma Irradiation Facility, Proc. of European Physical Society conference on High Energy Physics, 2017. 